\title{
Intracranial Metastasis in a Patient with Hepatocellular Carcinoma and Gastric Cancer
}

\author{
Akinobu Tawada $^{a}$ Tetsuhiro Chiba ${ }^{a}$ Yoshihiko Ooka $^{a}$ Hajime Yokota $^{b}$ \\ Naoya Kanogawa ${ }^{a}$ Tenyu Motoyama ${ }^{a}$ Tomoko Saito $^{a}$ \\ Sadahisa Ogasawara ${ }^{a} \quad$ Eiichiro Suzuki $^{\mathrm{a}}$ Naoyuki Hanari ${ }^{\mathrm{c}}$ \\ Hisahiro Matsubara ${ }^{c}$ Naokatsu Saeki ${ }^{d}$ Michiyo Kambe ${ }^{e}$ \\ Takashi Kishimoto $^{f}$ Yukio Nakatani $^{\mathrm{e}}$ Osamu Yokosuka ${ }^{a}$ \\ Departments of ${ }^{a}$ Gastroenterology and Nephrology, ${ }^{b}$ Radiology, ${ }^{\mathrm{c}}$ Frontier Surgery, \\ ${ }^{\mathrm{d}}$ Neurological Surgery, ${ }^{\mathrm{e}}$ Diagnostic Pathology, and ${ }^{\mathrm{f}}$ Molecular Pathology, Graduate School \\ of Medicine, Chiba University, Chiba, Japan
}

\section{Key Words}

Multiple primary malignancy · Hepatocellular carcinoma - Gastric cancer · Double cancer . Intracranial metastasis $\cdot$ Surgical resection

\begin{abstract}
A 76-year-old man was referred to our hospital with visual disturbance, weakness of the left upper and lower limbs, and gait disturbance. He had previously received transarterial chemoembolization for hepatocellular carcinoma (HCC) 3 and 10 years ago. When he had received radiofrequency ablation for HCC recurrence 2 years ago, total gastrectomy was also performed for his gastric cancer. Subsequently, sorafenib had been administrated for concomitant lung metastatic tumors. On admission, MRI revealed an intra-axial tumor with perifocal edema. The level of carcinoembryonic antigen, but not alpha-fetoprotein, markedly increased. The tumor was successfully removed by craniotomy and pathological examination revealed that it was composed of adenocarcinoma, which was consistent with the primary gastric cancer. After surgery, his neurological disturbances rapidly resolved. Additional gamma-knife treatment was also performed for another small brain metastasis detected after craniotomy. Subsequently, sorafenib administration was discontinued and S-1 was administered postoperatively. Successful treatment of intracranial metastasis of gastric cancer is important and meaningful, even in patients with multiple primary malignancies.
\end{abstract}


Tawada et al.: Intracranial Metastasis in a Patient with Hepatocellular Carcinoma and Gastric Cancer

\section{Introduction}

Hepatocellular carcinoma (HCC) is the sixth most common cancer worldwide, and it is the third most common cause of death from cancer [1]. Recent studies demonstrated clinical features of multiple primary malignancies involving HCC $[2,3]$. However, HCC patients bearing other malignancies with distal metastasis are not only uncommon, but also difficult to diagnose. Therefore, the therapeutic approach for and the clinical course of these patients remains unclear. Herein, we describe a case of metachronous multiple primary malignancies consisting of HCC and gastric cancer with intracranial metastasis.

\section{Case Report}

A 76-year-old man visited our hospital because of visual disturbance, weakness of the left upper and lower limbs, and gait disturbance. The patient had been treated for cirrhosis caused by chronic hepatitis C virus (HCV) infection. Because HCC was detected, approximately $60 \mathrm{~mm}$ in diameter, in the right lobe, he had undergone transarterial chemoembolization 3 and 10 years earlier. When radiofrequency ablation was performed for the recurrent HCC 2 years ago, gastric cancer was incidentally found (fig. 1a). Subsequently, he underwent gastrectomy with D1 lymphadenectomy and the tumor was successfully removed. Pathological examination revealed that the tumor was composed of moderately differentiated tubular adenocarcinoma. The staging of the lesion was T3N0. Additionally, multiple lung tumors were concurrently detected (fig. 1b). The patient had neither a history of smoking nor an elevation of tumor markers for primary lung cancer (such as squamous cell carcinoma antigen and neuron-specific enolase). Since the resected gastric cancer histologically lacked an involvement of lymph nodes, we considered the possibility that lung tumors were of HCC origin. Subsequently, sorafenib was administered. Because of adverse events such as a handfoot skin reaction, the dose of sorafenib was reduced from 800 to $200 \mathrm{mg} /$ day.

On admission, serum levels of hepatobiliary enzymes and renal function markers such as blood urea nitrogen and creatinine were normal. The serum level of ammonia was 91 $\mu \mathrm{g} / \mathrm{dl}$. Although blood count tests showed slight anemia (hemoglobin: $11.5 \mathrm{~g} / \mathrm{dl}$ ) and thrombocytopenia $\left(8.5 \times 10^{4} / \mu \mathrm{l}\right)$, his white blood cell count was normal $(4,000 / \mu \mathrm{l})$. AntiHCV antibody was positive, but hepatitis B surface antigen was negative. The level of carcinoembryonic antigen markedly increased to $44.1 \mathrm{ng} / \mathrm{ml}$.

However, the levels of alpha-fetoprotein (AFP), des-gamma-carboxy prothrombin (DCP), and carbohydrate antigen 19-9 (CA19-9) were within the normal range (5.6 ng/ml, 21 $\mathrm{U} / \mathrm{ml}$, and $10.2 \mathrm{U} / \mathrm{ml}$, respectively). Although the patient's consciousness level was normal [Glasgow Coma Scale was a full score (E4V5M6)], he exhibited left-sided hemifield visual disturbance and left-sided hemiparesis. Enhanced MRI revealed an intra-axial tumor in the right occipital cerebral lobe (fig. 2). Taking into consideration the markedly elevated level of carcinoembryonic antigen, but not AFP or DCP, we made a preoperative diagnosis of intracranial metastasis of gastric cancer, and a craniotomy of the tumor was performed. Macroscopic examination of the resected specimen revealed that the tumor was yellowishwhite, solid, and accompanied by necrosis (fig. 3a). The size of the tumor was $38 \times 30 \times 32$ $\mathrm{mm}$. Pathological examination revealed that the tumor was consistent with metastatic adenocarcinoma of gastric cancer origin (fig. $3 \mathrm{~b}$ ).

The postoperative course was uneventful. The patient's visual disturbance, hemiparesis, and gait disturbance markedly recovered to normal after surgery. Follow-up MRI conducted 1 week after the craniotomy revealed the successful removal of the tumor. Because a small 
Tawada et al.: Intracranial Metastasis in a Patient with Hepatocellular Carcinoma and Gastric Cancer

lesion was detected in the left precentral gyrus, the patient received additional gamma-knife treatment $(20$ Gy). Sorafenib administration was discontinued and S-1 (tegafur/gimeracil/oteracil potassium, $50 \mathrm{mg} /$ day) was administered postoperatively. He was alive for 5 months after the gamma-knife treatment, without any recurrence.

\section{Discussion}

Advances in diagnostic modalities and therapeutic approaches have improved the prognosis of patients with multiple primary malignancies. In the diagnosis of multiple primary malignancies, the widely accepted criteria were established by Warren et al. [4]. According to the criteria, each of the tumors must be distinct and present a definite picture of malignancy. The probability of one being a metastasis of the other must be excluded. It has been reported that the prevalence of multiple primary malignancies varies from 0.7 to $11.7 \%[5,6]$. In Western countries, prostate, colon, and lung cancers are the most commonly observed in multiple primary malignancies involving HCC [7]. In contrast, gastric cancer predominates in Asian countries [8, 9].

In our case, gastric cancer was detected 8 years after the first treatment of HCC. Although multiple lung metastases were observed concurrently, it was difficult to determine whether the tumor was derived from HCC or gastric cancer. We considered the possibility of HCC origin because of an increase in the serum level of AFP and PIVKA-II despite the curative treatment of recurrent HCC and histological findings of resected gastric cancer not involving the lymph nodes. Subsequently, sorafenib was administered.

Intracranial metastasis of gastric cancer is an extremely rare but critical condition. The frequency of intracranial metastasis in patients with gastric cancer ranges from 0.16 to $2.0 \%$ $[10,11]$. It has been documented that aggressive treatments such as resection and/or whole brain radiation therapy could improve the quality of life and prolong the survival $[12,13]$. In our case, resection of the intracranial tumor rapidly improved his neurological disturbances. Although another intracranial lesion was detected after craniotomy, the tumor was successfully treated with a gamma-knife. Given that the multiple lung tumors gradually enlarged during the administration of sorafenib, it was undeniable that the lung tumors were derived from gastric cancer. Eventually, the patient received postoperative adjuvant therapy with S-1. Together, these findings indicate the possibility that sufficiently aggressive treatment for intracranial metastasis of gastric cancer enables further anti-cancer treatment and improves the prognosis, even of patients with multiple primary malignancies.

When original tumors are radically treated in patients with multiple primary malignancies, further treatment including craniotomy and gamma-knife for the metastatic intracranial tumors might contribute to prolonged survival. Considering that intracranial metastasis of gastric cancers is frequently accompanied by bone, liver, and lung metastases [10,14], it is of importance to determine whether or not aggressive treatment, including surgical resection, improves both the performance status and the prognosis of the patients.

\section{Disclosure Statement}

The authors declare that they have no conflicts of interest. 


\section{Case Reports in Oncology}

\begin{tabular}{l|l}
\hline \multicolumn{2}{l}{ Case Rep Oncol 2014;7:199-203 } \\
\hline DOI: 10.1159/000360982 & $\begin{array}{l}\text { ○ 2014 S. Karger AG, Basel } \\
\text { www.karger.com/cro }\end{array}$ \\
\hline
\end{tabular}

Tawada et al.: Intracranial Metastasis in a Patient with Hepatocellular Carcinoma and Gastric Cancer

\section{References}

1 Ferlay J, Shin HR, Bray F, et al: Estimates of worldwide burden of cancer in 2008:GLOBOCAN 2008. Int J Cancer 2010;127:2893-2917.

-2 Zeng QA, Qiu J, Zou R, et al: Clinical features and outcome of multiple primary malignancies involving hepatocellular carcinoma: a long-term follow-up study. BMC Cancer 2012;12:148.

-3 Kai K, Miyoshi A, Kitahara K, et al: Analysis of extrahepatic multiple primary malignancies in patients with hepatocellular carcinoma according to viral infection status. Int J Hepatol 2012;2012:495950.

4 Warren S, Gates 0: Multiple primary malignant tumors: a survey of the literature and a statistical study. Am J Cancer 1932;16:1358-1414.

-5 Berge T, Cederqvist L, Schonebeck J, et al: Multiple primary malignant tumours. An autopsy study of a circumscribed population. Acta Pathol Microbiol Scand 1969;76:171-183.

6 Haddow AJ, Boyd JF: Multiple primary neoplasms in the Western Hospital Region, Scotland: a survey based on cancer registration data. Scott Med 1972;17:143-152.

-7 Fernández-Ruiz M, Guerra-Vales JM, Castelbón-Fernández FJ, et al: Multiple primary malignancies in Spanish patients with hepatocellular carcinoma: analysis of a hospital-based tumor registry. J Gastroenterol Hepatol 2009;24:1424-1430.

8 Takayasu K, Kasugai H, Ikeya S, et al: A clinical and radiologic study of primary liver cancer associated with extrahepatic primary cancer. Cancer 1992;69:45-51.

-9 Lin DY, Liaw YF, Wu CS, et al: Hepatocellular carcinoma associated with second primary malignancy. Liver 1987;7:106-109.

10 York JE, Stringer J, Ajani JA, et al: Gastric cancer and metastasis to the brain. Ann Surg Oncol 1999;6:771776.

11 Kim M: Intracranial involvement by metastatic advanced gastric carcinoma. J Neurooncol 1999;43:59-62.

12 Bartelt S, Momm F, Weissenberger C, et al: Patients with brain metastases from gastrointestinal tract cancer treated with whole brain radiation therapy: prognostic factors and survival. World J Gastroenterol 2004;10:3345-3348.

13 Go PH, Klaassen Z, Meadows MC, et al: Gastrointestinal cancer and brain metastasis: a rare and ominous sign. Cancer 2011;117:3630-3640.

14 Kasakura Y, Fujii M, Mochizuki F, et al: Clinicopathological study of brain metastasis in gastric cancer patients. Surg Today 2000;30:485-490.
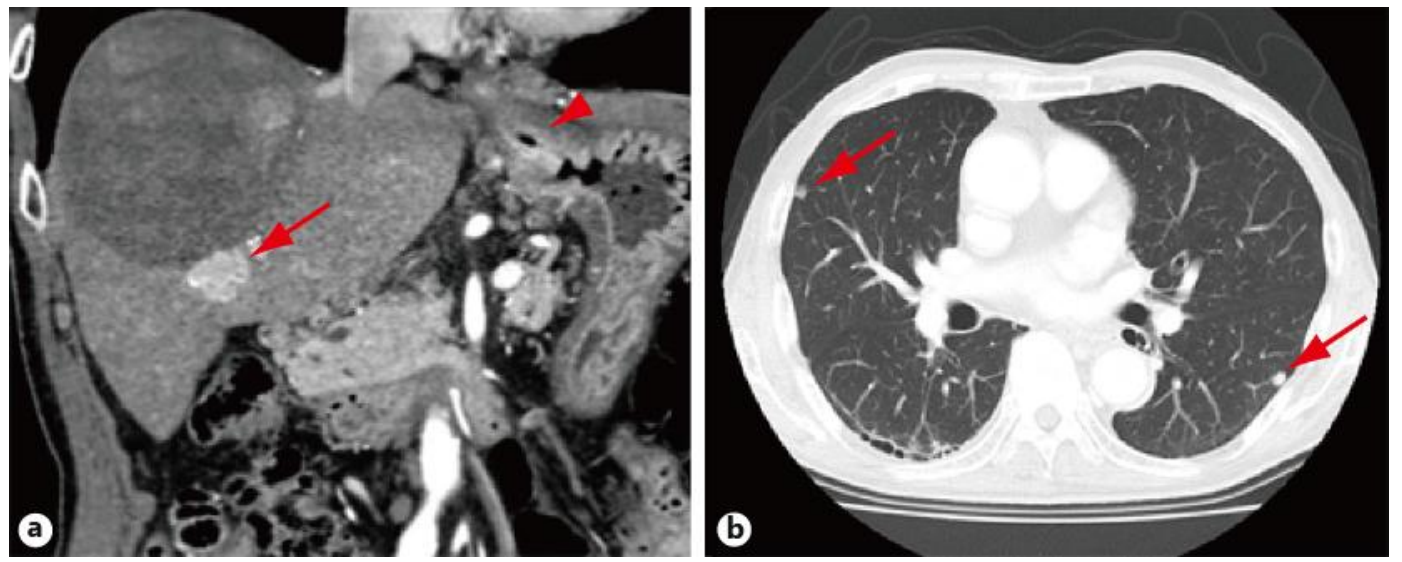

Fig. 1. CT findings. a Coronal contrast-enhanced CT in the arterial phase showing a recurrent hepatocellular carcinoma (arrow) and mucosal irregularity under the cardia, suggesting gastric carcinoma (arrowhead). b Multiple lung metastases (arrows) are depicted. 


\section{Case Reports in Oncology}

\begin{tabular}{l|l}
\hline Case Rep Oncol 2014;7:199-203 \\
\hline DOI: $10.1159 / 000360982$ & $\begin{array}{l}\text { C 2014 S. Karger AG, Basel } \\
\text { www.karger.com/cro }\end{array}$ \\
\hline
\end{tabular}

Tawada et al.: Intracranial Metastasis in a Patient with Hepatocellular Carcinoma and Gastric Cancer
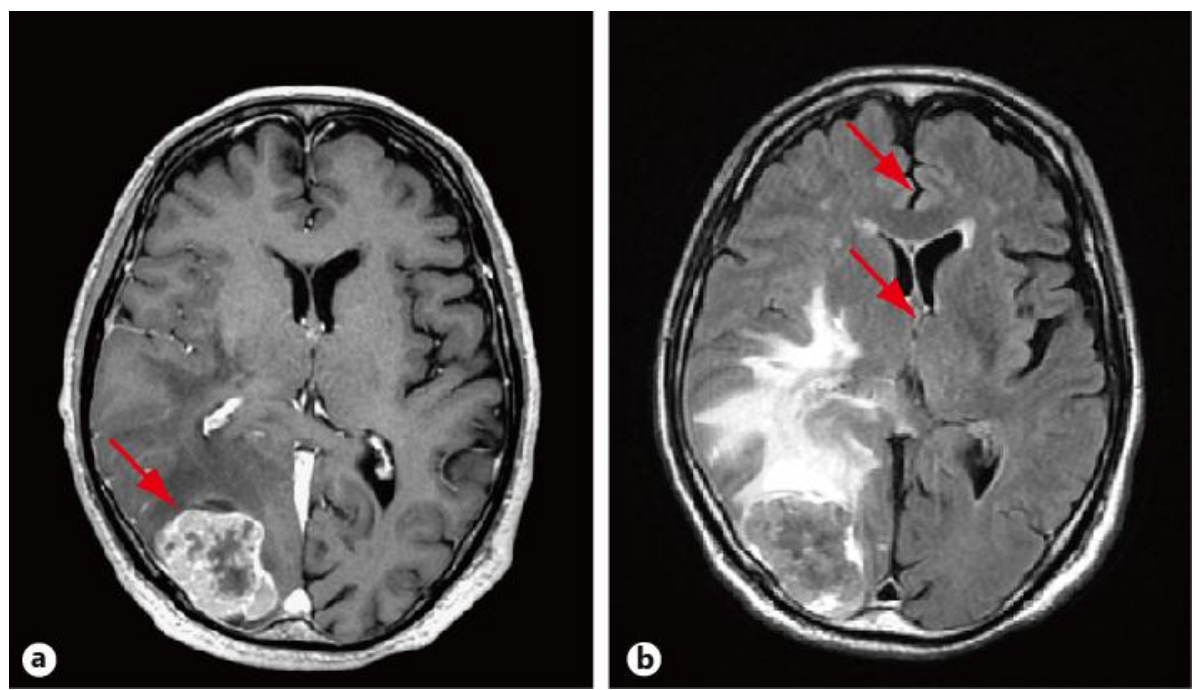

Fig. 2. MRI findings. a Contrast-enhanced T1-weighted image demonstrating a hyperintense tumor (arrow) with central necrosis. b T1-weighted FLAIR image showing a tumor with a hyperintense signal area, consistent with edema. The mass effect causes a midline shift (arrows).
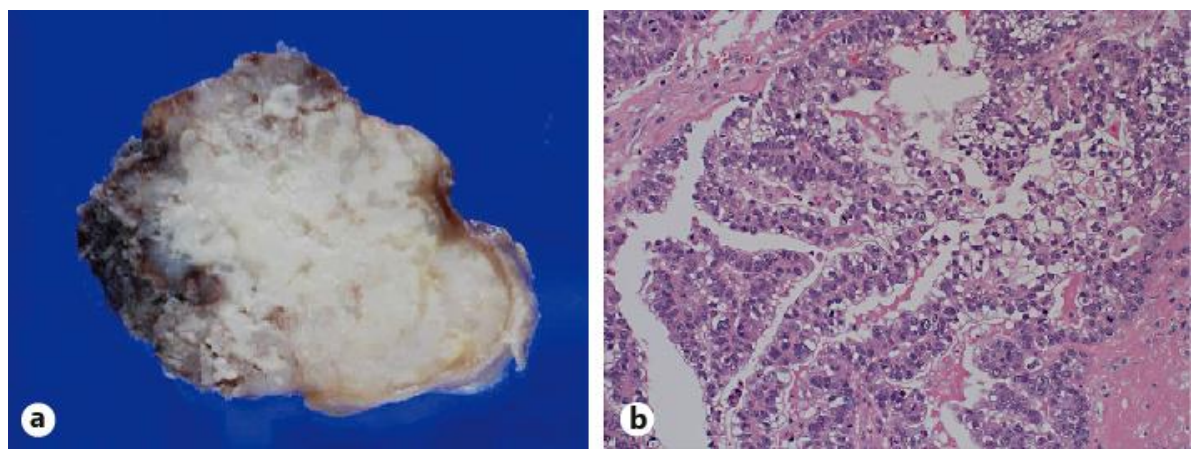

Fig. 3. Pathological examination of the intracranial tumor. a Macroscopic findings of the resected tumor. b HE staining of the tumor, $\times 20$. 